INPLASY

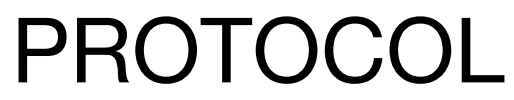

To cite: Zhang et al.

Monotherapy with a P2Y12

inhibitor or aspirin for

secondary prevention in

ischemic stroke: a systematic

review and meta-analysis.

Inplasy protocol 2020110131.

doi:

10.37766/inplasy2020.11.0131

Received: 28 November 2020

Published: 28 November 2020

Corresponding author:

Xiaoyuan Niu

niuxiaoyuan1958@163.com

Author Affiliation:

First Hospital of Shanxi

Medical University

Support: None.

Review Stage at time of this submission: Data analysis.

Conflicts of interest:

There are no conflicts of

interests.

\section{Monotherapy with a P2Y12 inhibitor or aspirin for secondary prevention in ischemic stroke: a systematic review and meta-analysis}

Zhang, K1; Wang, Y2; Niu, $X^{3}$.

Review question / Objective: The aim of this meta-analysis of randomized controlled trials is to compare the efficacy and safety of P2Y12 inhibitor and aspirin for secondary prevention in patients with ischemic stroke.

Condition being studied: Stroke is an enormous and serious public health problem. According to the World Health Organization, 15 million people suffer stroke worldwide each year. It is also a major cause of death and disability worldwide. Approximately $80 \%$ to $87 \%$ of all strokes are ischemic (that is due to a blockage of an artery in the brain) in white populations and about $67 \%$ in Asian populations.

INPLASY registration number: This protocol was registered with the International Platform of Registered Systematic Review and Meta-Analysis Protocols (INPLASY) on 28 November 2020 and was last updated on 28 November 2020 (registration number INPLASY2020110131).

\title{
INTRODUCTION
}

Review question / Objective: The aim of this meta-analysis of randomized controlled trials is to compare the efficacy and safety of P2Y12 inhibitor and aspirin for secondary prevention in patients with ischemic stroke.
Condition being studied: Stroke is an enormous and serious public health problem. According to the World Health Organization, 15 million people suffer stroke worldwide each year. It is also a major cause of death and disability worldwide. Approximately $80 \%$ to $87 \%$ of 
all strokes are ischemic (that is due to a blockage of an artery in the brain) in white populations and about $67 \%$ in Asian populations.

\section{METHODS}

Search strategy: We searched PubMed, Embase, and the Cochrane Central Register of Controlled Trials for studies published from inception to July 2020. Reference list of all selected articles will independently screened to identify additional studies left out in the initial search.

Participant or population: Patients with recent ischemic stroke within the previous year will be included.

Intervention: P2Y12 inhibitors monotherapy (any dosage) for at least four weeks.

Comparator: Aspirin monotherapy (any dosage) for at least four weeks.

Study designs to be included: Only randomized controlled trials

Eligibility criteria: Eligible studies were those that compared P2Y12 inhibitor with aspirin monotherapy for secondary prevention in patients who experienced ischemic stroke in the previous year. Trials with more than two groups for which a subset of interventions satisfied the inclusion criteria were kept in the analysis after having discarded the groups that did not satisfy the inclusion criteria. We excluded studies comparing dual versus single antiplatelet therapy, studies assessing anti-thrombotic agents different from aspirin or P2Y12 inhibitors, and studies not reporting clinical outcomes. We also excluded studies with less than 1 month of active comparison between single antiplatelet therapy strategies, studies with less than 25 patients, and those with overlapping populations (eg, studies reporting prespecified or post-hoc analyses). No limits were set for age and other comorbidities. Only studies published in English were included for review of the full text.
Information sources: PubMed, Embase, and the Cochrane Central Register of Controlled Trials

Main outcome(s): 1. a new stroke event (ischemic or hemorrhagic). 2. a new clinical vascular event (ischemic stroke, hemorrhagic stroke, myocardial infarction, or vascular death), analyzed as a composite outcome and also as individual outcomes. 3. all cause death.

Quality assessment / Risk of bias analysis: The risk of bias was assessed using the revised Cochrane risk-of-bias tool (RoB 2). Two investigators (Kaili Zhang, Yongle Wang) independently assessed five domains of bias for each outcome: (1) randomisation process, (2) deviations from intended interventions, (3) missing outcome data, (4) measurement of the outcome, and (5) selection of the reported results.

Strategy of data synthesis: Odds ratios (ORs) and $95 \%$ Cls were calculated using the Der Simonian and Laird random-effects model, with the estimate of heterogeneity being taken from the Mantel-Haenszel method. The number of patients needed to treat to prevent one event was calculated from weighted estimates of pooled ORs from the random-effects meta-analytic model. The presence of heterogeneity among studies was measured with Cochran's $Q x^{2}$ test, with a $p$ value of up to 0.10 considered significant, and to measure consistency we used the $I^{2}$ test. An $I^{2}$ value of $0 \%$ indicates no observed heterogeneity, and larger values indicate increasing heterogeneity. $I^{2}$ values of up to $25 \%$ indicate low heterogeneity, up to $50 \%$ indicate moderate heterogeneity, and above $50 \%$ indicate high heterogeneity.

Subgroup analysis: Pre-specified subgroup analyses included the efficacy outcomes and bleeding endpoints according to the presence of potential trial-level effect modifiers - the type of P2Y12 inhibitor used (clopidogrel, ticlopidine or ticagrelor) the estimated risk of bias (ie, low risk of bias vs some concerns for risk of bias),race, aspirin dosage, time to index event from 
enrolment. Post-hoc subgroup analyses included an analysis according to the presence of ipsilateral large-artery atherosclerotic stenosis. ORs with $95 \% \mathrm{Cls}$ for the co-primary endpoints were reported in each subgroup.

Sensibility analysis: Pre-specified sensitivity analyses were performed with the use of a fixed-effects model, and by iteratively removing one study at a time to confirm that our findings were not driven by any single study. Post-hoc sensitivity analyses included an analysis which removed all studies using ticlopidine as P2Y12 inhibitor. All analyses were performed according to the intention-totreat principle. The two-sided level of significance was denoted as $p$ below 0.05 . Statistical analyses were done with Stata (version 13.1; Stata Corp, College Station, TX, USA).

Language: Only English.

Country(ies) involved: China.

Keywords: P2Y12 inhibitor; aspirin; monotherapy; secondary prevention; ischemic stroke; meta-analysis.

Contributions of each author:

Author 1 - Kaili Zhang - The author conceived and designed the study, assessed studies for possible inclusion and drafted the manuscript.

Email: zhangkaili-2009@163.com

Author 2 - Yongle Wang - The author collected and analysed the data.

Email: yonglewang@163.com

Author 3 - Xiaoyuan Niu - The author conceived and designed the study.

Email: niuxiaoyuan1958@163.com 